\title{
Ensemble-based active learning for class imbalance problem
}

\author{
Yanping Yang, Guangzhi Ma
}

School of Computer Science and Technology; Huazhong University of Science and Technology, Wuhan, China.

Email: maguangzhi.hust@gmail.com

Received 8 September 2010; revised 20 September 2010; accepted 25 September 2010

\begin{abstract}
In medical diagnosis, the problem of class imbalance is popular. Though there are abundant unlabeled data, it is very difficult and expensive to get labeled ones. In this paper, an ensemble-based active learning algorithm is proposed to address the class imbalance problem. The artificial data are created according to the distribution of the training dataset to make the ensemble diverse, and the random subspace re-sampling method is used to reduce the data dimension. In selecting member classifiers based on misclassification cost estimation, the minority class is assigned with higher weights for misclassification costs, while each testing sample has a variable penalty factor to induce the ensemble to correct current error. In our experiments with UCI disease datasets, instead of classification accuracy, F-value and G-means are used as the evaluation rule. Compared with other ensemble methods, our method shows best performance, and needs less labeled samples.
\end{abstract}

Keywords: Class Imbalance, Active learning, Ensemble, Random Subspace, Misclassification Cost

\section{INTRODUCTION}

In the medical diagnosis, it is common that there is a huge disproportion in the number of cases belonging to different classes [1]. For example, the number of cancer cases is much smaller than that of the healthy. The traditional classifiers, however, are incapable of countering such class imbalance problem, because they favor the majority class. Moreover, the minority class is much more important in real applications. In addition, in real world, there are abundant unlabeled data but labeled instances are difficult, time-consuming or expensive to obtain. It will in turn make the labeled minority class much fewer further, which often degrades the performance of traditional classifiers greatly. As a result, active learning with unlabeled imbalanced data becomes an important issue in machine learning [3].

To address the class imbalance problem, the direct way is to reduce the imbalance by re-sampling original dataset. Some methods try to under-sampling majority class, like Tomek link [4], condensed nearest neighbor rule [5] and neighborhood cleaning rule [6][7]. In these methods, the majority samples in certain area are considered as useless and can be removed from training dataset. But, there is a risk of missing representative samples. Other methods, like SMOTE [8], try to oversampling the minority class. In SMOTE method, the artificial datasets are created according to the distribution of the minority class. However, the enhancement will be little, if the created artificial datasets have the same properties as the labeled samples

Finding proper classifier for minority class is another way to counter class imbalance problem. Joshi [9] once modified the Boosting algorithm by assigning the minority class with a weight different from that of the majority class. Akbni [10] adjusted the SVM's decision-boundary by modifying the kernel function. But, the certain classifier is only efficient in countering specific class imbalance, and cannot be extended to other applications. Another trend is to use ensemble of classifiers, which often has better performance than single classifier. But, the performance depends on the diversity of the ensemble [11]. If classifiers in an ensemble have the same property, there will be less improvement of performance even with more classifiers.

Active learning techniques are conventionally used to solve problems where there are abundant unlabeled data but rare labeled ones [3]. Recently, various approaches on active learning from imbalanced datasets have been proposed in literatures [12]. For instance, as a good classifier, support vector machine (SVM) was proposed in active learning for the imbalance problem [14]. To reduce the computational complexity in dealing with large imbalanced datasets, this method was implemented in a random set of training populations, instead of the entire training dataset. In [16], bootstrap-based over- sampling was proposed to reduce the imbalance in the application 
of word sense disambiguation. Facing the class imbalance issue, however, both re-sampling and classifier strategy have their own advantage as well as disadvantage. The best way is to combine them together [17]. But progress in this field is little.

In this paper, an ensemble-based active learning with artificial samples is proposed to address class imbalanced problem by using unlabeled data. Different from random sampling, we try to use active selection strategy to label the sample with potential benefit to the ensemble's diversity. In addition, we will create artificial datasets from the distribution as the training dataset. The conversely labeling of each artificial data will bring diversity to the ensemble. Both the training dataset and the artificial dataset will be re-sampled according to random subspace concept. It will release the difficulty of traditional sampling methods while facing with high-dimension data. Further, when choosing member classifiers according to misclassification cost, the minority class is assigned with a higher weight for misclassification cost, and each testing sample has a variable penalty factor to induce the ensemble to correct current error. In the experiments with UCI disease datasets, instead of accuracy, F-value and G-mean are used to evaluate the performance, since they are better for minority classification tasks.

The rest of this paper is organized as follows. In Section 2, the proposed ensemble is described in detail, including the creation of artificial datasets, random subspace re-sampling and misclassification cost estimation. Section 3 introduces how to implement active learning with our proposed ensemble method. In experiment part, the new evaluation rules are introduced. Based on experiments on the UCI datasets, our proposed method is compared with other state-of-art methods.

\section{RANDOM SUBSPACE ENSEMBLE WITH ARTIFICIAL DATASETS}

In our ensemble-based active learning, the ensemble algorithm is the core. So, in this section, we will introduce our Random Subspace Ensemble with Artificial Data (RSEAD) in detail.

\subsection{Overview}

Figure 1 is the algorithm of our Random Subspace Ensemble with Artificial Data (RSEAD). Each member classifier in the ensemble is created via the iteration steps in Figure 1.

At the beginning of the algorithm, the training dataset $T$ will be mapped into another dataset $T$, in a $m$-dimension subspace. Then a classifier will be created based on $T$, and used to initiate the ensemble $C^{*}$. Also, the misclassification cost of current ensemble will be calculated. Whereafter, the algorithm will enter follow- ing iteration:

1) According to the distribution of the training dataset $T$, an artificial dataset will be generated. The size of the artificial dataset will be in a certain ratio, Rsize, to that of training dataset. They will be labeled with a class different from what the ensemble predicts

2) In the $m$-dimension subspace, both $T$ and $R$ will be re-sampled to $T$, and $R$,

3) A new classifier $C$, will be learned from both labeled $R$, and $T$,. In order to guarantee the performance of the ensemble when pursuing the diversity, the misclassification cost of the new ensemble with $C$, is calculated. Compared with the previous ensemble, if the new classifier brings more misclassification cost, it will be removed; otherwise, it will be kept in the ensemble;

4) The above steps will be iterated until algorithm returns the expected size of ensemble, or the number of iterations reaches the limited value.

To predict the class of an unlabeled sample $x$, each member classifier $C_{i}$ in ensemble $C$, will assign $x$ with an membership probability, $\hat{P}_{c, y}(x)$. Then the ensemble will calculate membership probability of each class $y$ for sample $x$ via following equation:

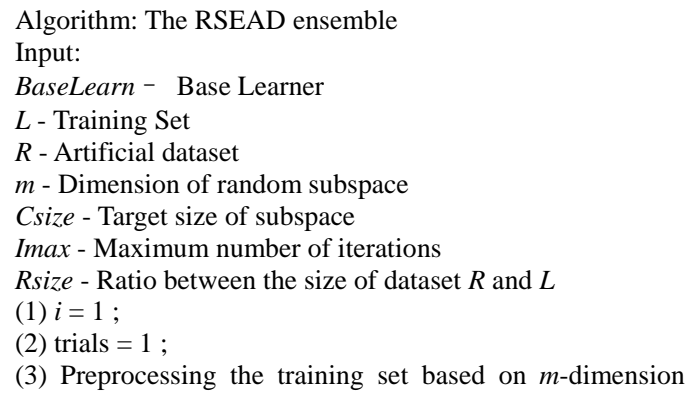

(3) Preprocessing the training set based on $m$-dimension subspace : $T^{\prime}=R S M-\operatorname{sampling}(T)$
(3) $C_{i}=$ BaseLearn $\left(T^{\prime}\right)$
(4) $C^{*}=\left\{C_{i}\right\}$
(5) Calculate the ensemble error, $\varepsilon ; i=i+1$
(6) While $i<$ Csize and trials $<$ Imax
\{
(7) Create artificial dataset, the size will be Rsize $\times|T|$;

(8) Assign each artificial sample a label different from $C^{*}$ 's prediction.

(9) Re-sample the training set and artificial set in m-dimension subspace:

$T^{\prime}=R S M-\operatorname{sampling}(T) \quad R^{\prime}=R S M-\operatorname{sampling}(R)$

(10) $T^{\prime}=T^{\prime} \cup R^{\prime}$

(11) $C_{i}=$ BaseLearn( $\left(T^{\prime}\right) \quad C^{*}=C^{*} \cup\left\{C_{i}\right\}$

(12)Calculate the misclassification cost of new ensemble, $\varepsilon^{\prime}$

(13) If $\varepsilon^{\prime} \leq \varepsilon$ then $\left\{\varepsilon=\varepsilon^{\prime}, i=i+1\right\}$

(14) else $\left\{C^{*}=C^{*}-\left\{C^{\prime}\right\}\right.$; trials $=$ trials +1$\}$

Figure 1. Algorithm of RSEAD ensemble. 


$$
\hat{P}_{y}(x)=\frac{\sum_{C_{i} \in C^{*}} \hat{P}_{C_{i}, y}(x)}{\left|C^{*}\right|}
$$

Equation (1) reflects the probability of $x$ belonging to class $y$. Therefore, the label with largest membership probability will be assigned to $x$ :

$$
C^{*}(x)=\arg \max _{y \in Y} \hat{P}_{y}(x)
$$

\subsection{Creation and Labeling of Artificial Datasets}

The diversity is a critical factor for a successful ensemble [11]. An ensemble will have less diversity if its member classifiers have the same property. To bring more diversity, Bagging [19] divides the training set into several smaller one, while Boosting adjusts the distribution of the training dataset according to the chosen classifier [20]. Further, in Random Forest [21], both training dataset and feature space are divided into smaller ones to train different classifiers. However, all these methods depend on the training dataset to induce the diversity. Therefore, if the training dataset is not big enough, the diversity will be limited.

In our active learning method, the RSEAD ensemble's diversity will be guaranteed in three ways: 1) with active learning, the large pool of unlabeled data can be sampled to get good training datasets; 2) besides the training dataset, the artificial data are also created for training classifier; 3) both the original training and the artificial datasets will be re-sampled in subspace to enhance diversity. In this part, we will focus on the creation of artificial dataset and their labeling.

In our method, the artificial data are created by randomly picking data points from an approximation of the training dataset distribution. The numeric attributes are defined according to the mean and the standard deviation of the training dataset, and generated in Gaussian distribution. For a nominal attribute, its value is based on the probability of the occurrence of each distinct value in its domain. The Laplace smoothing is used if a certain nominal attribute is absent in the training dataset. Further, to construct an artificial data, there is a simplifying assumption that the attributes are independent, because it will cost much time and labeled data to accurately estimate the joint probability distribution of these attributes.

In each iteration shown in Figure 1, the ensemble will predict the class label for each artificial data $x$. Firstly, ensemble will give a membership probability of $x$ belonging to certain class $y$. The zero membership probability will be replaced by a small non-zero value in case that it may act as a denominator. Then the artificial data will be labeled a class that is different from what the ensembles predict. Therefore, if current ensemble pre- dicts the probability of $x$ belonging to $y$ is $\hat{P}_{y}(x)$, then, the choice of label for $x$ will be based on $\hat{P}_{y}^{\prime}(x)$ :

$$
\hat{P}_{y}^{\prime}(x)=\frac{1 / \hat{P}_{y}(x)}{\sum_{y} 1 / \hat{P}_{y}(x)}
$$

Let us show this labeling method with a two-class problem. For instance, for an artificial sample $x$, the ensemble estimates that it has $20 \%$ probability of being a positive sample and $80 \%$ probability of being a negative one. In other words, the ensemble believes that $x$ is more likely a negative sample. In our method, to create a new classifier with more diversity, $x$ will be assigned with a positive label, and then used to train a new classifier.

The ensemble often has higher accuracy than single member classifier if each member classifier is not related with others. Therefore, our method of labeling artificial data can reduce the relevancy between classifiers, which will in turn bring the ensemble with higher accuracy and less generalization error.

\subsection{Re-Sampling in Subspace}

Re-sampling is the popular way to deal with class imbalance problem. However, most of sampling methods, like SMOTE, often work in the whole feature space, which is not efficient in countering high-dimension datasets. In addition, they often try to consider the class imbalance and the properties of the dataset as a whole. The data, however, often exhibit characteristics and properties at a local level, rather than the global level. Hence, it is important to study the dataset in a reduced subspace. Although a certain feature subspace may only lead to a weak classifier, the ensembling of such weak ones can make a strong classifier [22], since it induces higher diversity, which is an important condition for a classifier with good performance.

To this end, we proposed the Random-SubspaceMapping Sampling (RSM-sampling) algorithm.

Suppose we have a dataset $L$, which has a $n$-dimension space: $|L|=1, \quad F=\left\{F_{1}, F_{2}, \ldots, F_{n}\right\}$. Any data $P \in L$ can be represented as $P=\left\{P_{1}, P_{2}, \ldots, P_{n}\right\}$, where $P_{i}$ is the value of the related feature $F_{i}$ in the feature space $F$.

If the dimension of each subspace is set to $m, m<n$, the number of the likely subspace will be $k_{\max }=C_{n}^{m}$. When $m=[n / 2], k_{\max }$ has its biggest value. For our algorithm, each feature subspace will bring a candidate classifier. We often choose Cszie $<k_{\max }$ classifiers to construct an ensemble, since not every candidate classifier will help enhance the ensemble,s performance.

Before re-sampling, a subspace $S$ should be randomly selected from the feature space $F .|F|=m<n$. $S=\left\{S_{1}, S_{2}, \ldots, S_{m}\right\} \subset F$. Then, in the feature subspace, 
each data $P \in L$ will be mapped into

$$
P_{S}=\left\{P_{s 1}, P_{s 2}, \ldots, P_{s m}\right\} \text {. }
$$

In each iteration step of our algorithm, both the training dataset $L$ and the artificial dataset $R$ are re-sampled in chosen sub-space.

\subsection{Misclassification Cost Estimation}

When pursuing the diversity, the performance of ensemble should be guaranteed too. To address the problem of class imbalance, the misclassification cost is used to replace the traditional classification error. A new classifier will be kept in the ensemble if it helps decrease the misclassification cost; otherwise, it will be removed.

In our algorithm, the minority class is assigned with a higher weight of misclassification cost than that of the majority class. Also, each test sample will be assigned with a penalty factor. If current ensemble makes wrong decision on it, its penalty factor will be increased; otherwise, its penalty factor will be decreased. In this way, the ensemble will choose the new classifier that helps to correct the error of current ensemble. Also, since the minority samples have more chance to be misclassified, this penalty factor will bring an ensemble proper for minority class.

Suppose we have $t$ samples to evaluate the ensemble based on misclassification cost. Firstly, each sample's penalty factor will be initialized as:

$$
d_{i}^{1}=1 / t \quad 1 \leq i \leq t
$$

The misclassification cost of the ensemble gotten in $k$-th iteration can be represented as:

$$
\varepsilon_{k}=\sum_{i=1}^{t} \cos t\left(y_{i}, C_{k}^{*}\left(x_{i}\right)\right) \times d_{i}^{k}
$$

In Equation (5), $y_{i}$ is the correct class label of testing sample $x_{i}, C_{k}^{*}\left(x_{i}\right)$ is the predicted class of the ensemble for $x_{i} . d_{i}^{k}$ is $x_{i}$,S penalty factor for the $k$-th iteration. $\cos t\left(y_{i}, C_{k}^{*}\left(x_{i}\right)\right)$ is the weight of misclassifying a sample with label $y_{i}$ as class $C_{k}^{*}\left(x_{i}\right)$. When $y_{i}=C_{k}^{*}\left(x_{i}\right)$, there is $\cos t\left(y_{i}, C_{k}^{*}\left(x_{i}\right)\right)=0$ because classification is correct.

If the misclassification cost in the $k$-th iteration is less that that in the $(K-1)$-th iteration, then newly created classifier will be kept in ensemble. There, we have the coefficient of performance enhancing:

$$
\alpha_{k}=\ln \left(\left(1-\varepsilon_{k}\right) / \varepsilon_{k}\right) / 2
$$

Each testing sample's penalty factor will be modified according to current ensemble's prediction. If current ensemble makes correct classification on $x_{i}$, its penalty factor $d_{i}^{k+1}$ will be decreased to:

$$
d_{i}^{k+1}=d_{i}^{k} \exp \left(-a_{k}\right)
$$

Otherwise, it will be increased to:

$$
d_{i}^{k+1}=d_{i}^{k} \exp \left(a_{k}\right)
$$

Please note, all samples, new penalty factors will be normalized as following:

$$
\begin{gathered}
Z_{k+1}=\sum_{i=1}^{t} d_{i}^{k+1} \\
d_{i}^{k+1}=d_{i}^{k+1} / Z_{k+1} .
\end{gathered}
$$

$d_{i}^{k+1}$ will be used in the $(k+1)$-th iteration.

The design of misclassification cost weigh $\cos t\left(y_{i}, C_{k}^{*}\left(x_{i}\right)\right)$ and penalty factor $d_{i}^{k+1}$ will help the ensemble to pick the classifiers that can better deal with minority class.

\section{ACTIVE LEARNING WITH THE ENSEMBLE RSEAD}

The ensemble with diversity will be used in active learning for selecting unlabeled data. Like the QBC [23], our proposed active learning method also chooses the unlabeled samples that have the biggest prediction difference among the classifiers in the ensemble. Such prediction difference is often called uncertainty, which is calculated via margin measure in our algorithm. The margin is defined as the difference of membership probability between the samples most likely class and second most likely class.

$$
\operatorname{Margin}\left(C^{*}, x\right)=\hat{P}_{y 1}(x)-\hat{P}_{y 2}(x)
$$

where $y 1$ and $y 2$ are class labels of unlabeled sample $x$ predicted by ensemble $C^{*} . y 1$ has the highest membership probability for $x$, while $y 2$ is the second highest one. Then, the uncertainty can be represented as:

$$
\operatorname{Uncertainty}\left(C^{*}, x\right)=\frac{1}{\operatorname{Margin}\left(C^{*}, x\right)+\delta}
$$

where the $\delta$ is a small value in case margin is 0 . The smaller margin is, the bigger the uncertainty is. For a two-class task, when $\hat{P}_{y 1}(x)=\hat{P}_{y 2}(x)$, the margin will be 0 , and $x$ will have the biggest uncertainty, Uncertainty $\left(C^{*}, x\right)=1 / \delta$

\section{EXPERIMENTS}

To evaluate our method,s effectiveness for medical diagnosis, eight disease datasets from the UCI machine learning repository [24] are used in experiments. In this section, we will discuss the experiments in detail.

\subsection{Evaluation Rule}

In a two-class task, a classifier will have four kinds of prediction results [25] for dataset with $N$ samples, shown in Table 1. TP and $F N$ responsively mean the number of correctly and wrongly classified positive samples, while 
$T N$ and $F P$ mean the number of correctly and wrongly classified negative samples.

The classification accuracy is often calculated as :

$$
\text { Accuracy }=(T P+F N) / N \text {. }
$$

The accuracy rule, however, is not a good one for imbalance classification [26], for example, if there are only $1 \%$ positive samples but $99 \%$ negative samples. Simply classifying all samples as negative class will bring 99\% accuracy, but misclassified $1 \%$ positive samples will bring enormous cost. Therefore, such $99 \%$ accuracy is a disaster for medical diagnosis.

In our proposed method, F-value [27] defined in Equation (13) is used to evaluate the classifier for imbalance class problem.

$$
F-\text { value }=\frac{\left(1+\beta^{2}\right) \times \text { Precision } \times \text { Recall }}{\beta^{2} \times \text { Precision }+ \text { Recall }}
$$

where, Precision $=T P /(T P+F N)$; Recall $=T P /(T P+F P)$. $\beta$ measures the importance of Precision vs. Recall. In our method, $\beta=1$, which means Precision and Recall is equally important.

In addition, G-Mean [28] is also used in evaluating the performance of our classifier.

G-mean $=\sqrt{\text { PositiveAccuracy } \times \text { NegativeAccuracy }}$

where PossitiveAccuracy = Precision, NegativeAccuracy $=T N /(T N+F P)$. It can be seen that G-mean measure tries to build a balance between positive class and negative class.

\subsection{Datasets Description}

For testing, eight disease datasets from UCI are chosen. Some basic information about them is summarized in Table 2, in which P:N means the number of positive samples via number of negative samples.

\subsection{Experiments on the Dimension of Subspaces}

As discussed in 2.3, to randomly select a $m$-dimension subspace from a $n$-dimension feature space, the number of choices will be $k_{\max }=C_{n}^{m}$. In our algorithm, the $m$ is recommended as $m=[n / 2]$, since it bring the maxim choice. Even if we choose a Csize $<K_{\max }$, bigger value of $k_{\max }$ means more chance to get good member classifiers.

Based on dataset Breast-w, we test the relation between the dimension of a subspace and the performance of a classifier based on F-value. The result is shown in Figure 2. In this experiment, the Csize of the ensemble is 30. Since Breast- $W$ has 9 features, $m=1$ and $m=9$ are meaningless to this experiment. So, the dimension of feature space $m$ will be changed from 2 to 8 in experiment. In Figure 2, F-value will reach its peak when $m=$ 5. The F-value at $m=4$ is a little less than $m=5$, al- though they have the same $k_{\max }$. The reason may be that 5 -feature subspace brings more information than 4 -feature one. From Figure 2, we can see that if $m$ is too small, the information in each subspace is too little to train a good classifier; but if $m$ is too big, there will be little diversity among different subspace, which is also bad for the performance of the ensemble. This experiment shows that $m=[n / 2]$ is a good setting for dataset Breast-W.

\subsection{Experiment on the Size of Ensemble}

In this experiment, we test the relation between the ensemble's size and its classification performance. The Breast- $W$ is still used and the result is shown in Figure 3.

Table 1. Classification of a two-class problem.

\begin{tabular}{llll}
\hline & $\begin{array}{l}\text { \#classified as } \\
\text { positive }\end{array}$ & $\begin{array}{l}\text { \#classified as } \\
\text { negative }\end{array}$ & Total \\
\hline Positive sample & $T P$ & $F N$ & $T P+F N$ \\
Negative sample & $F P$ & $T N$ & $F P+T N$ \\
Total & $T P+F P$ & $F N+T N$ & $N$ \\
\hline
\end{tabular}

Table 2. Summary of experimental UCI disease datasets.

\begin{tabular}{llll}
\hline Dataset & \#features & \#instances & P: $\mathbf{~}$ \\
\hline Colic & 22 & 368 & $136: 232$ \\
Sick & 30 & 3772 & $231: 3541$ \\
Diabetes & 8 & 768 & $268: 500$ \\
SAheart & 11 & 462 & $160: 302$ \\
Hepatitis & 20 & 155 & $32: 123$ \\
mammograph & 5 & 961 & $445: 516$ \\
Breast-W & 9 & 699 & $241: 458$ \\
Spect & 22 & 267 & $55: 212$ \\
\hline
\end{tabular}

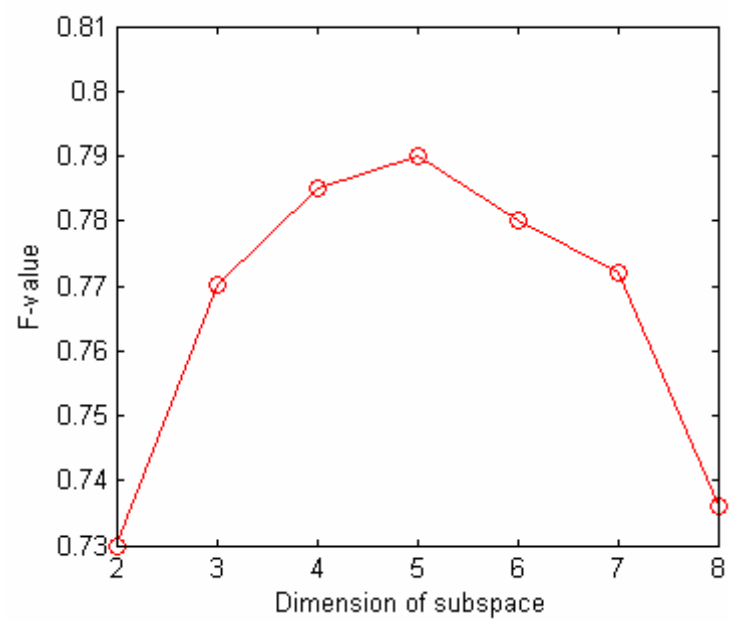

Figure 2. F-value for different dimension of subspace on Breast- $W$ dataset. 


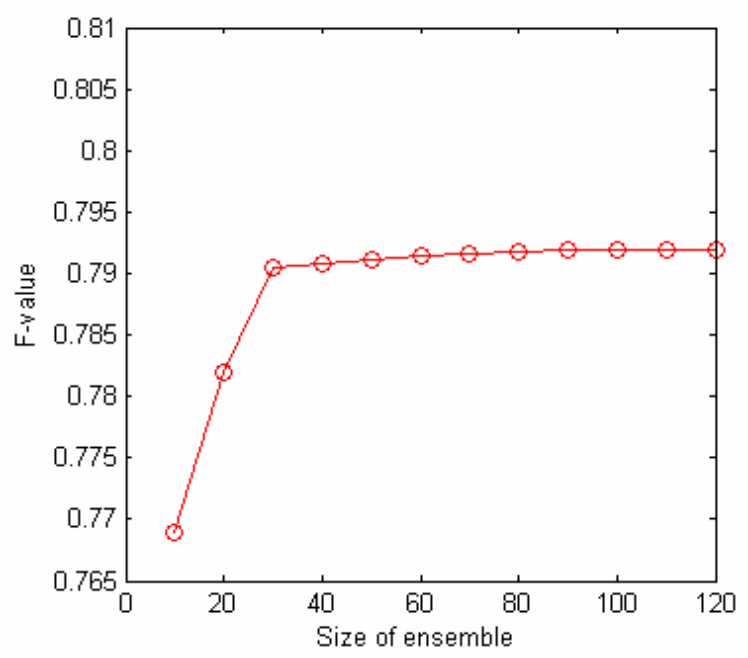

Figure 3. F-Value for different size of an ensemble on Breast- $W$ dataset.

In the experiment, the dimension of subspace is fixed as $m=[n / 2]=[9 / 2]=5$. Therefore, there will be $C_{9}^{5}=126$ choices of subspaces to train Csize classifiers for the ensemble. In Figure 3, the F-value increases quickly when Csize grows from 10 to 30 , but the enhancement is not big when Csize is changed from 30 to 120. It shows that for dataset Breast- $W, 30$ subspaces with 5 dimensions are enough to build a good ensemble. The additional subspace will contribute little to the diversity of ensemble, and there will be no much enhancement in performance, though the computation cost grows much. Therefore, 30 is a trade-off between performance and computation cost for dataset Breast-W.

\subsection{Experiment Result}

In this experiment, we firstly test the performance of our proposed RSEAD ensemble algorithm. For comparison, two state-of-art classification algorithms, Bagging and Adaboost, are chosen. For fair comparison, C4.5 is used as base learner, and is configured with the default setting in Weka [29]. In the evaluation of performance, F-value and G-mean are used in experiments with 10-fold cross validation. For RSEAD algorithm, it has a setting with $m=[n / 2], k=30$, and $\operatorname{Imax}=50$.

Shown in Table 3 is the F-value for the minority class in each dataset, while Table 4 is the G-value for every whole dataset. For each dataset, the highest value is marked in bold. For convenience of comparison, the base learner C4.5 is also used as the reference. In the tables, Ada represents the Adaboost algorithm.

In Table 3 and 4., all 3 ensembles have good F-value and G-mean than C4.5 on eight datasets. Compared with Bagging and Adaboost, our RSEAD has higher F-value and G-mean on most of dataset. From Table 3, it can be concluded that RSEAD has the best performance for minority class on 6 datasets. On dataset mammograph, the difference between ensembles is not significant. The reason may be that ratio between the minority and majority classes is near $4: 5$, which has a very small class imbalance. Also, dataset mammograph is defined only by 5 features, which leaves little room for our random subspace re-sampling method to enhance the ensemble's performance. In the evaluation based on G-mean, our RESEAD wins for all 8 datasets. From Tables 3 and 4, it can be seen that our ensemble RESEAD has better performance than Bagging and ADABOOST in countering problem of imbalance class. This advantage comes from the unique way of creating each member classifier as well as the misclassification cost based decision in selecting proper classifiers. Compared with Bagging, Adaboost has better performance, because Adaboost introduces different cost weight for different misclassification. It also indirectly proves the correctness of our misclassification cost estimation.

To further test the performance of our active learning method with RSEAD ensemble, the Bagging and Adaboost are also merged into the active learning architecture for comparison. Single RSEAD is tested further as reference. Table 5 shows how many samples each algorithm needs to get certain F-value on each dataset. Compared with RSEAD, the active learning methods need fewer samples to get the same F-value. Among the three active learning methods, our Active-RSEAD has significant advantage, which benefits from the design of RSEAD ensemble.

Table 3. F-vaule for minority class in each dataset.

\begin{tabular}{lcccc}
\hline Dataset & C4.5 & RSEAD & Bagging & Ada \\
\hline Colic & 76.54 & $\mathbf{8 0 . 9 7}$ & 79.71 & 80.03 \\
Sick & 87.65 & $\mathbf{9 3 . 2 3}$ & 90.44 & 91.43 \\
Diabetes & 61.4 & $\mathbf{7 1 . 8}$ & 67.9 & 69.8 \\
SAheart & 55.3 & $\mathbf{7 5 . 2}$ & 67.4 & 73.1 \\
Hepatitis & 52.8 & 68.4 & 67.2 & $\mathbf{6 8 . 5}$ \\
mammograph & 79.5 & 81.2 & 82.1 & $\mathbf{8 3 . 2}$ \\
Breast-W & 89.7 & $\mathbf{9 5 . 6}$ & 92.3 & 94.0 \\
Spect & 73.1 & $\mathbf{7 9 . 7 6}$ & 76.6 & 77.5 \\
\hline
\end{tabular}

Table 4. G-mean for each dataset.

\begin{tabular}{lrrrc}
\hline Dataset & C4.5 & RSEAD & Bagging & \multicolumn{1}{c}{ Ada } \\
\hline Colic & 81.5 & $\mathbf{8 5 . 5}$ & 83.4 & 84.51 \\
Sick & 91.2 & $\mathbf{9 5 . 8}$ & 95.6 & 95.2 \\
Diabetes & 64.3 & $\mathbf{7 6 . 4}$ & 71.4 & 74.3 \\
SAheart & 60.4 & $\mathbf{7 7 . 8}$ & 72.3 & 77.5 \\
Hepatitis & 58.4 & $\mathbf{7 6 . 3}$ & 74.3 & 73.4 \\
mammograph & 88.4 & $\mathbf{8 9 . 4}$ & 89.1 & 89.3 \\
Breast-W & 94.3 & $\mathbf{9 6 . 5}$ & 95.3 & 95.4 \\
Spect & 82.3 & $\mathbf{8 5 . 6}$ & 82.4 & 83.4 \\
\hline
\end{tabular}


Table 5. Number of sampling for target F-vlalue.

\begin{tabular}{|c|c|c|c|c|c|}
\hline Dataset & RSEAD & Active-RSEAD & Active-Bagging & Active Adaboost & Target F-value \\
\hline Colic & 41 & 23 & 35 & 37 & $85 \%$ \\
\hline Sick & 321 & 134 & 178 & 165 & $93 \%$ \\
\hline Diabetes & 245 & 101 & 114 & 106 & $75 \%$ \\
\hline SAheart & 280 & 123 & 157 & 167 & $60 \%$ \\
\hline Hepatitis & 117 & 45 & 56 & 54 & $95 \%$ \\
\hline mammograph & 100 & 24 & 35 & 30 & $80 \%$ \\
\hline Breast-W & 32 & 36 & 45 & 75 & $95 \%$ \\
\hline Spect & 53 & 38 & 43 & 39 & $75 \%$ \\
\hline
\end{tabular}

\section{CONCLUSIONS}

To address the problem of imbalance class in medical diagnosis, an ensemble-based active learning method is proposed. Our ensemble algorithm, RSEAD, introduces the subspace sampling method to reduce the complexity of computation and bring more diversity together with the creation of artificial datasets. Further, in evaluating the quality of each classifier candidate based on misclassification cost, the minority class is assigned with a higher weight for misclassification costs, while each testing sample has a variable penalty factor to induce the ensemble to correct current classification error.

In above experiments, eight UCI disease datasets are chosen. The F-value and G-mean are used instead of classification accuracy to evaluate the performance of classifiers. The result shows that our proposed ensemble method has better performance than others. Moreover, in active learning experiment, having the same performance with F-value rule, our method needs fewer samples. These experiments show that our ensemble-based active learning method has significant advantage than traditional methods.

Ensemble-based active learning is a promising method to counter the problem of class imbalance in medical diagnosis. But, there are still many issues for further studying. For example, our method only deals with two-class tasks, while the real world has many multi-class tasks. In addition, the noise in a dataset is not considered in current study. Also, the weighting method in our method needs further improvement from both theory and implementation. Therefore, we will focus on these issues to improve our active-RSEAD method in following research work.

\section{REFERENCES}

[1] Japkowicz, N. and Stephen, S. (2002) The class imbalance problem: A systematic study. Intelligent Data Analysis, 6(5), 203-231.

[2] Gustavo, E.A., Batista, P.A., Ronaldo, C., et a1. (2004) A study of the behavior of several methods for balancing machine learning training data. SIGKDD Explorations, 6(1), 20-29.

[3] Settles, B. (2009) Active Learning Literature Survey. Computer Sciences Technical Report 1648, University of Wisconsin-Madison.

[4] Tomek, I. (1976) Two modifications of CNN. IEEE Transaction on Systems Man and Communications, 6, 769-772.

[5] Hart, P.E. (1968) The condensed nearest neighbor rule. IEEE Transaction on Information Theory, 14(3), 515516.

[6] Laurikkala, J. (2001) Improving identification of difficult small classes by balancing class distribution. Proceedings of the 8th Conference on AI in Medicine, Cascais, Portugal, Europe: Artificial Intelligence Medicine, 63-66.

[7] Wilson, D.L. (1972) Asymptotic properties of nearest neighbor rules using edited data. IEEE Transaction on Systems, Man and Communications, 2(3), 408-421.

[8] Chawlan, V., Bowyer, K.W. and Hall, L.O. (2002) SMOTE: Synthetie minority over-sampling technique. Journal of Aflificial Intelligence Research, 16(1), 321357.

[9] Joshi, M., Kumar, V. and Agarwal, R. (2001) Evaluating boosting algorithms to classify rare classes: Comparison and improvements. Proceedings of the 1st IEEE International Conference on Data Mining. Washington DC: IEEE Computer Society, 257-264.

[10] Akbani, R., Kwek, S. and Japkowicz, N. (2004) Applying support vector machines to imbalanced datasets. Proceedings of the 15th European Conference on Machines Learning, Pisa, Italy, 39-50.

[11] Krogh, A. and Vedelsby, J. (1995) Neural network ensembles, cross validation and active learning. Advances in Neural Information Processing Systems, 7, 231-238.

[12] Provost, F. (2000) Machine learning from imbalanced data sets 101. Invited paper for the AAAI, Workshop on Imbalanced Data Sets, Menlo Park, CA.

[13] Abe, N. (2003) Invited talk: Sampling approaches to learning from imbalanced datasets: Active learning, cost sensitive learning and beyond. ICML-KDD Workshop: Learning from Imbalanced Data Sets.

[14] Ertekin, S., Huang, J. and Giles, C.L. (2007) Active learning for class imbalance problem. Proceedings of Annual International ACM SIGIR Conference Research and development in information retrieval, Amsterdam, Netherlands, 823-824.

[15] Ertekin, S., Huang, J., Bottou, L. and Giles, C.L. (2007) Learning on the border: Active learning in imbalanced 
data classification. Proceedings of the sixteenth ACM conference on Conference on information and knowledge management, November 6-8, Lisboa, Portugal, 127-136.

[16] Zhu, J. and Hovy, E. (2007). Active Learning for Word Sense Disambiguation with Methods for Addressing the Class Imbalance Problem. In Proc. Joint Conf. Empirical Methods in Natural Language Processing and Computational Natural Language Learning, Prague, 783-790.

[17] Chawla, N.V., Lazarevic, A. and Hall, O. (2003) SMOTEBoost: improving prediction of the minority class in boosting: knowledge discovery in databases. Proceeding of the 7th European Conference on Principles and Practice of Knowledge Discovery in Databases. Cavtat Dubrovnik, 107-119.

[18] Veropoulos, K., Campbell, C. and Cristianini, N. (2009) Con- trolling the sensitivity of support vector machines. Proc of Intemational Joint Confbrence on AI, 55-60.

[19] Breiman, L. (1996) Bagging predictors. Machine Learn -ing, 24(2), 123-140.

[20] Abe, N. and Mamitsuka, H. (1998) Query learning strategies using boosting and bagging. Proceedings of the International Conference on Machine Learning (ICML), Morgan Kaufmann, 1-9.

[21] Breiman, L. (2001) Random forests. Machine Learning, 2001, 45(1), 5-32.
[22] Kleinberg, E.M. (1990) Stochastic discrimination. Annals of Mathematics and Artificial Intelligence, 1(1-4), 207239.

[23] Seung, H.S., Opper, M. and Sompolinsky, H. (1992) Query by committee. In Proceedings of the ACMWorkshop on Computational Learning Theory, 287-294.

[24] Blake, C., Keogh, E., and Merz, C.J. UCI repository of machine learning databases. http://www.ics.uci.edu

[25] Su, C.T., Chen, L.S. (2006) Knowledge acquisition through information granulation for imbalanced data. Expert Systems with applications, 31, 531-541.

[26] Joshi, M. (2002) On evaluating performance of classifiers for rare classes. Proceeding of the 2nd IEEE International Conference on Data Mining, Maebishi, Japan, 641-644.

[27] Kotsiantis, S., Kanellopoulos, D., Pintelas, P. (2006) Handling imbalanced datasets: A review. GESTS International Transactions on Computer Science and Engineering, 30(1), 25-36.

[28] Guo, H., Viktor, H. (2004) Learning from imbalanced data sets with boosting and data generation: the DataBoost-IM approach. Sigkdd Explorations, 6(1), 30-39.

[29] Witten, I. H., Frank, E. (2005) Data mining-pracitcal machine learning tools and techniques with JAVA implementations. 2nd Edition, Morgan Kaufmann Publishers. 